\title{
Optimizing patient throughput in nuclear medicine: a semi-quantitative tool for scheduling bone scintigraphy
}

\author{
Claudine Als
}

Published online: 10 October 2007

(C) Springer-Verlag 2007

Dear Sir,

A busy nuclear medicine department may be defined by a high patient throughput and a high number of data acquisitions in relation to the number of gamma cameras at disposal. Hence, optimizing camera occupation while preserving constant quality standards is mandatory. This is especially true in case the unit is part of a hospital setting, where appointment planning for out-patients has to face up every day with urgent demands concerning hospitalized patients. As in a small country such as Luxembourg, where the nuclear medicine market is commercially speaking insignificant, another reality that dedicated computer programmes tailored to our national needs and covering administrative as well as scheduling purposes do unfortunately not exist.

The problem in scheduling is that some scintigraphic procedures require a constant camera acquisition time per patient independently of the clinical indication and that some others do not. Protocols with a rather stable acquisition time per patient are, for instance myocardial perfusion, thyroid uptake and imaging, pulmonary ventilation and perfusion, static and dynamic renal scans. Appointment schedules are then rather easily organized. The situation is, however, different with bone scintigraphy, as camera acquisition time varies substantially with the

C. Als $(\bowtie)$

Department of Nuclear Medicine, Clinique Ste Thérèse,

36 , rue Ste Zithe,

2763 Luxembourg, Grand Duchy of Luxembourg

e-mail: claudine.als@cst.lu

C. Als

Institute of Clinical Chemistry, University of Bern, Inselspital, 3010 Bern, Switzerland clinical indication, spanning theoretically from some minutes to some hours. Therefore, scheduling constant acquisition times per bone scan would result in undesired patient waiting as well as in blank camera periods. As bone scintigraphy is a frequent exam in any department of nuclear medicine, the question is quite relevant. We have enhanced the efficiency of scheduling bone scintigrams with a semi-quantitative approach.

In our department, all scintigraphic orders ('ordonnances') by referring physicians, and especially orders for bone scans, are evaluated by the medical doctor (MD) specialist in nuclear medicine before attributing an appointment to the patient. Depending on the clinical indication, the MD decides upon the necessity of mono- vs bi-sequential acquisitions, of early images, of late phase planar and/or SPECT images. To describe the scheduled camera sequences per bone scan, we developed a standardized semi-quantitative code (Tables 1 and 2) with the following aims:

1. to have the cameras busy as much as possible per working day and to avoid blank periods,

2. to facilitate communication within the medico-technical team concerning patient scheduling and throughput,

3. to have patients wait as little as possible in the waiting room upon arrival,

4. to provide patient appointments with a delay as short as possible.

The semi-quantitative code consists of two numbers. The first number defines the necessity or not of an early (vascular) phase acquisition (Table 1). The second number roughly defines the duration of late phase acquisitions (Table 1). Some clinical examples are given in Table 2.

From a practical point of view and according to Tables 1 and 2: 
Table 1 A semi-quantitative code derived from the indication for bone scintigraphy roughly defines the scheduled duration of camera acquisition per patient

Definition of the bi-numbered code for bone scintigraphy $(\times-\times)$

\begin{tabular}{ll}
\hline $\begin{array}{l}\text { First number (defines necessity } \\
\text { or not of early/vascular phase) }\end{array}$ & $\begin{array}{l}\text { Second number (indicates rough } \\
\text { duration of late/osseous phase } \\
\text { acquisition) }\end{array}$ \\
\hline $0=$ no early phase acquisitions & $1= \pm 15$ minutes \\
required; $1=$ with early phase & $\begin{array}{l}2= \pm 30 \text { minutes } \\
3= \pm 45 \text { minutes } \\
\text { acquisition(s) }\end{array}$ \\
& $4= \pm 60$ minutes \\
\hline
\end{tabular}

1. the MD allocates the relevant semi-quantitative code to the bone scan order,

2. the reception team is thereafter able to efficiently allocate patient appointments related to the available cameras,

3. the camera programme is more efficiently 'filled up' due to standardized communication and thereby patient throughput is increased,

4. as an example, late phase images of three consecutive patients with an injury of the foot may, for instance be acquired on one camera while within a similar time span, images of a patient with a set-up for polyarthritis are acquired on another camera,

5. if necessary, in case of an equivocal or uncertain situation, additional acquisitions may be ordered at the end of the scheduled protocol and after clinical examination, so that the system is flexible,
6. the code is probably as well applicable to a nuclear medicine unit with one as with several gamma cameras,

7. in a unit with more than one camera, last-minute flexibility is nevertheless higher, as additional images may be acquired on another camera than the one in use for the initially scheduled acquisitions,

8. be it only for patients coming late for their appointment or cancelling their exam at the last minute, the system anyway has to deal with delays and, thus anyway, is flexible.

In conclusion, and in absence of a dedicated national computer programme, we tried to resolve the scheduling problems of our medico-technical team, generated by highly variable acquisition times with bone scans, spanning from some minutes to hours (Table 2). We therefore invented and implemented the above-described standardized code. The code is of course free of charge. Introduction and acceptance of the code in our department gave no problems. The higher the part of bone scans performed with a 'non-basic' protocol (i.e. basic protocol=whole body late images), the higher the impact of timing on fluidity of patient throughput. Camera planning and patient throughput became more convenient and more efficient. Patient satisfaction increased due to lesser waiting times. Feeling of stress within the team greatly decreased. All concerned actors-patients, staff and MDs-considered these improvements as relevant and as highly satisfying. We recommend this convenient and cheap tool to any busy department of nuclear medicine interested in efficiency and in an improved cost-benefit relation.

Table 2 Practical clinical examples of the bi-numbered code applied to bone scans in our department

\begin{tabular}{|c|c|c|c|c|}
\hline $\begin{array}{l}\text { Clinical indications } \\
\text { (examples) }\end{array}$ & $\begin{array}{l}\text { Vascular phase } \\
\text { images necessary? }\end{array}$ & $\begin{array}{l}\text { Osseous phase, type of image } \\
\text { acquisitions and scheduled } \\
\text { duration (min) }\end{array}$ & $\begin{array}{l}\text { Total duration } \\
\text { osseous } \\
\text { phase (min) }\end{array}$ & $\begin{array}{l}\text { Bi-numbered } \\
\text { code }\end{array}$ \\
\hline Lumbar backpain & (yes) & $\begin{array}{l}1 \text { static image }\left(5^{\prime}\right) \\
\left.\text { Lumbar SPECT ( } 20^{\prime}\right)\end{array}$ & $25-30$ & $1-2$ \\
\hline Loosening of hip prosthesis? & Yes & $\begin{array}{l}1 \text { static image }\left(5^{\prime}\right) \\
\text { Hip-femur SPECT (20') }\end{array}$ & $25-30$ & $1-2$ \\
\hline Osseous metastases? & None & Whole body planar (20’) & $20-25$ & $0-2$ \\
\hline Algodystrophy? & Yes & 3-5 static images of limb (à 5') & $15-30$ & $1-1(2)$ \\
\hline Injury of foot? & Yes & 2-3 static images of feet/ankles (à 5') & $10-20$ & $1-1$ \\
\hline Polyarthritis or other rheumatic condition? & Yes & $\begin{array}{l}\left.\text { Whole body planar ( } 20^{\prime}\right) \\
\left.\text { Feet+hands/wrists ( } 4 \text { images à } 5{ }^{\prime}\right)\end{array}$ & 45 & $1-3$ \\
\hline Spondylarthritis? & Yes & $\begin{array}{l}\left.\text { Whole body planar ( } 20^{\prime}\right) \\
\text { Feet+hands/wrists ( } 4 \text { images à } 5 \text { ') } \\
\text { Thoraco-lumbar SPECT }\left(20^{\prime}\right)\end{array}$ & $>6$ & $1-4$ \\
\hline
\end{tabular}

The scheduled duration includes patient installation/handling under the camera. Thereby patient throughput and efficiency of the department are increased. 